\title{
Large area radio frequency plasma for microelectronics processing
}

\author{
Z. Yu and D. Shaw \\ Colorado State University, Fort Collins, Colorado 80523 \\ P. Gonzales \\ NCR Microelectronics, Fort Collins, Colorado 80525 \\ G. J. Collins \\ Colorado State University, Fort Collins, Colorado 80523
}

(Received 5 October 1994; accepted 21 November 1994)

\begin{abstract}
Radio-frequency (rf) inductively coupled planar plasma (ICP) provides a better way to generate spatially confined high density gas discharge plasmas for microelectronics processing. Commercial processing equipment using this technique is currently available, but is limited in size to $20 \mathrm{~cm}$ in diameter by problems with plasma uniformity and antenna dielectric window erosion. We have developed a new planar ICP antenna and dielectric window design that allows for larger dimensions (up to $50 \mathrm{~cm}$ in diameter) with good uniformity. The current art ICP antenna requires a thick quartz (or ceramic) plate vacuum window to separate the rf inductor and the plasma. The larger the antenna diameter the thicker the dielectric. The thick dielectric reduces inductive coupling efficiency. The large area coil and associated matching network can introduce plasma uniformity problems. Our device incorporates both the rf inductor and the dielectric window inside the vacuum chamber, allowing space for a thin layer of quartz or other dielectric material. Thus, the dielectric window design is only focused on materials for the given process chemistry to be placed between the coil and the excited plasma, rather than also including mechanical strength to hold a vacuum over a wide area. This thin dielectric layer with our newly designed planar coil coupler allows the plasma to be scaled to a $50 \mathrm{~cm}$ diameter while maintaining radial uniformity. In this article we report only plasma ashing results for a $20 \mathrm{~cm}$ planar ICP device that shows $2 \%$ nonuniformity across $15 \mathrm{~cm}$ wafers. The measured flux of atomic oxygen and atomic hydrogen generated by a larger planar ICP scale device is also presented. (c) 1995 American Vacuum Society.
\end{abstract}

\section{INTRODUCTION}

Our present ICP source is based on prior work by IBM and Lam Research Corporation for plasma processing machines $^{1}$ and our own experiences with large area plasma lamps. $^{2-4}$ Commercial ICP processing still has several major unsolved problems and here we focus on wide area plasma uniformity and undesired antenna window erosion caused by the plasma. Our present design employs a unique vacuum and antenna structure with a novel matching circuit to achieve larger area planar ICP plasmas with good spatial uniformity. Photoresist ashing serves as a test vehicle for our studies. The rf power did not exceed $200 \mathrm{~W}$ in our experiments due to a power supply limitation. Nevertheless, due to better coupling, results rival those at $\mathrm{kW}$ power levels.

A thick window was used in prior works ${ }^{1,5,6}$ to inductively couple rf power into the process chamber, both to provide mechanical support of the vacuum required and to reduce capacitive coupling from the $\mathrm{rf}$ coil. In the case of diameters of quartz or alumina ceramic $20 \mathrm{~cm}$ or larger, the dielectric window must be at least $3 \mathrm{~cm}$ thick to support the difference in pressure between vacuum and atmosphere. This minimum thickness causes the following problems: (1) the cost of such large dimension dielectric materials is already high and becomes prohibitive above $50 \mathrm{~cm}$ diameter; and (2) the additional $3 \mathrm{~cm}$ antenna-to-plasma distance reduces the inductive coupling efficiency. Compared to our 6-mm-thick dielectric, a factor of at least 5 is lost requiring higher rf power input.

\section{EXPERIMENTS}

\section{A. Thin antenna dielectric liner}

We moved the rf coil into the vacuum chamber to exceed the limitations of the prior art. By doing this, it was not necessary to have a thick dielectric window. Instead, the rf coupling coil was molded into the vacuum with a dielectric inbetween the thin dielectric window and the coil. For example, EP 30 AO (Master Bond) epoxy with added alumina powder can be used since it has good thermal conductivity, and its thermal expansion coefficient is close to that of the ceramic liner on the rf antenna. Inside the process chamber between the rf coil and the plasma region, in the front of the epoxy encapsulated rf coil, a thin layer of liner material, such as quartz or high purity alumina ceramic as required by the process, may be laminated onto the coil assembly. Figure 1 shows this design. Note that the vacuum wall lies physically behind the coil to support the required vacuum seal. There is no pressure difference applied to the thin dielectric antenna window; therefore the liner layer needs only about a $6 \mathrm{~mm}$ thickness for chemical stability of the processing and to withstand plasma erosion. The replacement cost of a $6 \mathrm{~mm}$ quartz window is 10 times less than that of a $3 \mathrm{~cm}$ thick one.

\section{B. Low inductance coupler}

When one scales up the rf antenna diameter, an undesired high quality factor, $Q$, results due to the increase of stovetop coil inductance. High $Q$ reduces the efficiency of inductive 


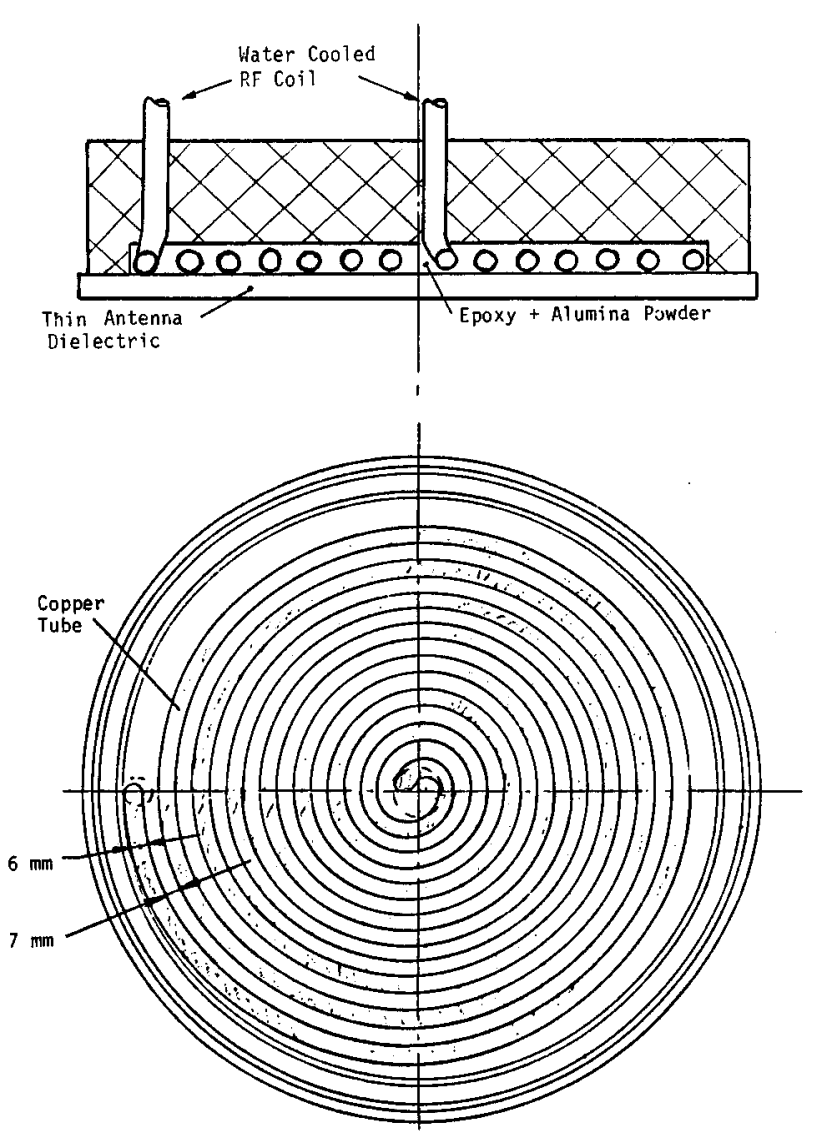

FIG. 1. Schematic diagram of the laminated rf coupler coil assembly with a thin dielectric antenna window.

coupling. ${ }^{7}$ Moreover, as described by Hopwood, ${ }^{6}$ the radial component of the rf magnetic field is not uniform over the radius of the coupling coil as size increases. It needs to be locally tailored along the radius.

We helped solve both high $Q$ and poor radial uniformity problems by dividing the large coupling coil into multisegments in series electrically as shown in Fig. 2. Each segment has a variable capacitor for individual tuning of that radial

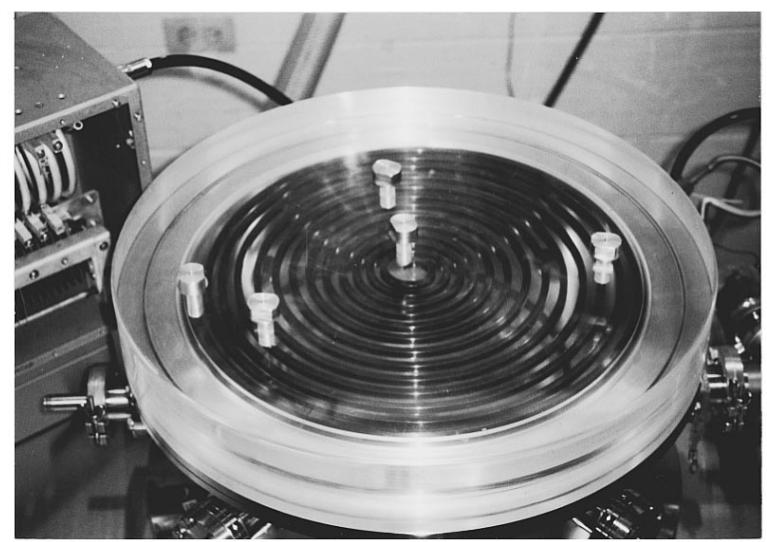

FIG. 2. The 40-cm-diam rf coupler coil with metal plugs dividing the coil into three segments. The tunable capacitors for separately tuning each segment are not shown.

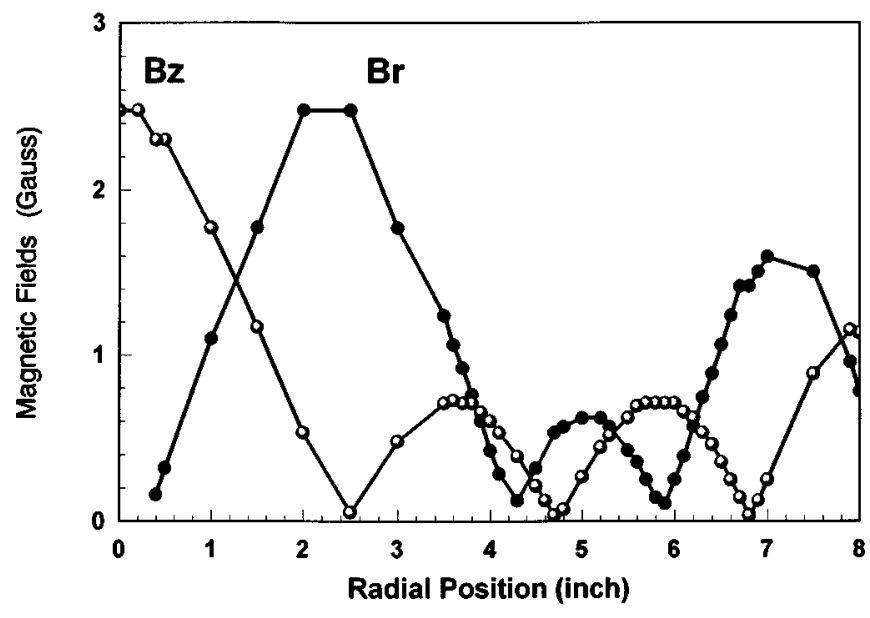

FIG. 3. Radial variations of $\mathrm{Br}$ and $\mathrm{Bz}$ components measured $3.8 \mathrm{~cm}$ below the coupler coil in the absence of a plasma.

section. The rf is totally self-coupled within these series segments and the inductance, $L$, of each $L-C$ resonance circuit is small enough to avoid high $Q$ operation. Moreover, the $Q$ value of each of the segments can be adjusted to control the resonance current within the subsegment circuit. As a result we tailored the radial component of the rf magnetic field induced in the plasma. Typical measured rf magnetic fields in the absence of a plasma are shown in Fig. 3. A second design uses a planar Archimedes spiral, $r(\theta)=a \theta$, coil with large ratio factor, " $a$," to reduce the number of turns for the coil covering the large area. To compensate for the nonuniform $\mathbf{B}$ field caused by the bigger space between coils of such a design, we use a multispiral structure. For example, a triple spiral can be described as

$$
\begin{aligned}
& \rho_{1}\left(\theta_{1}\right)=\left(\theta_{1} / \pi\right)+1, \\
& \rho_{2}\left(\theta_{2}\right)=\left(\theta_{2} / \pi\right)+1 / 3, \\
& \rho_{3}\left(\theta_{3}\right)=\left(\theta_{3} / \pi\right)-1 / 3 .
\end{aligned}
$$

The distinct electrical circuit for powering the Archimedes spiral of three associated coupling coils is shown in Fig. 4. The input rf power is transmitted to one of $L C$ resonances and couples efficiently to the other two resonances via mutual inductance between them.

\section{Plasma results}

Planar plasmas, excited by the planar spiral coil couplers from 20 to $50 \mathrm{~cm}$ in diameter, were examined in oxygen and hydrogen feedstock gas with regard to both their atomic radical flux versus input rf power [Fig. 5(b)] and their radical uniformity across the diameter [Fig. 5(a)]. The typical flux of ICP generated atomic oxygen, measured by a microbalance with a silver coated quartz sensor, ${ }^{8}$ was $10^{18}$ atoms $\mathrm{cm}^{-2} \mathrm{~s}^{-1}$ at the substrate location, $5.7 \mathrm{~cm}$ from the planar coupler at $100 \mathrm{~W}$ rf power at $13 \mathrm{MHz}$, as shown in Fig. 4. The atomic hydrogen flux under similar conditions was 10 times less. The atomic hydrogen detection employed a silver oxide coated microbalance sensor. ${ }^{9}$ The flux values are comparable 


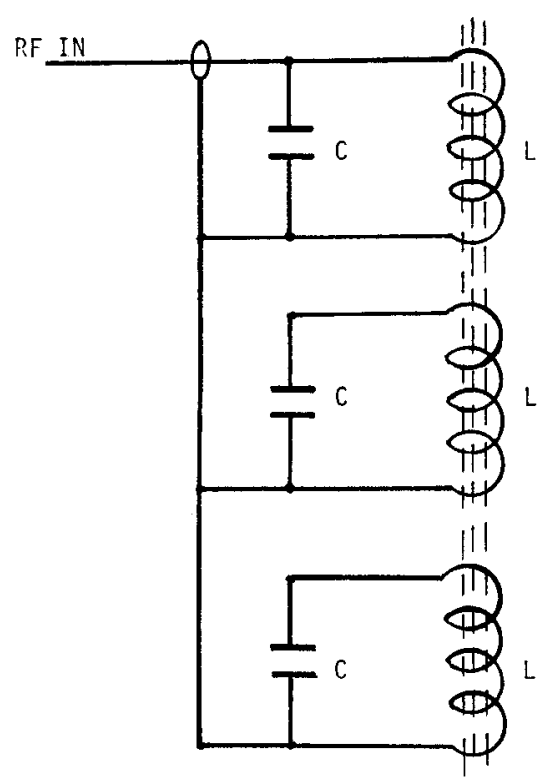

FIG. 4. Three units of $L C$ resonance linked via mutual inductance.
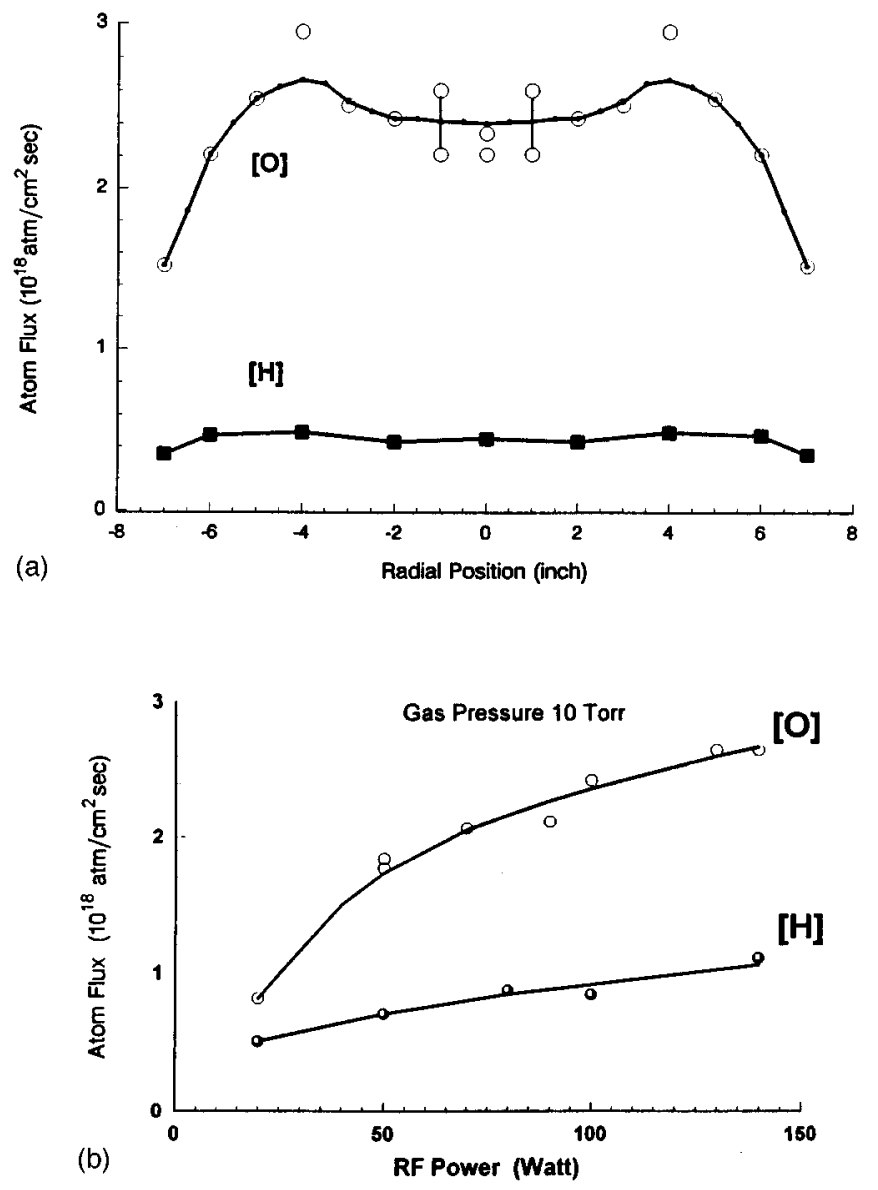

FIG. 5. (a) The atomic flux profile of both oxygen and hydrogen over a 40 $\mathrm{cm}$ rf coupler measured by a quartz crystal microbalance method developed by some researchers (Ref. 9). (b) The flux of atomic oxygen and hydrogen vs $\mathrm{rf}$ power in the center.

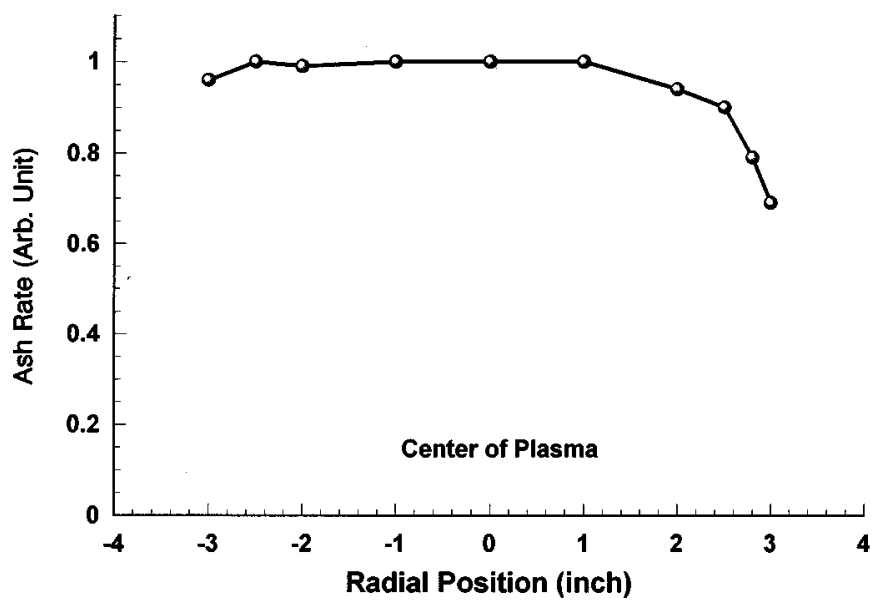

FIG. 6. Photoresist ash rate profile of a $20 \mathrm{~cm}$ coupler shows $2 \%$ deviation across the $15 \mathrm{~cm}$ center portions of the wafers.

to our prior direct current $(\mathrm{dc})$ discharge plasma in oxygen. ${ }^{8}$ In Fig. 5 we display the measured relative atomic hydrogen radical flux profile versus input rf power.

Photoresist ashing has been tested using a downstream prototype equipped with this new planar ICP apparatus. Ashing tests with Shipley 1400 positive photoresist result in an ash rate nonuniformity of $\pm 2 \%$ over a 15 -cm-diam substrate, as shown in Fig. 6.

\section{Other related studies}

To better compare the ICP to prior work ${ }^{2}$ the following gives our best results with modified designs of our earlier work.

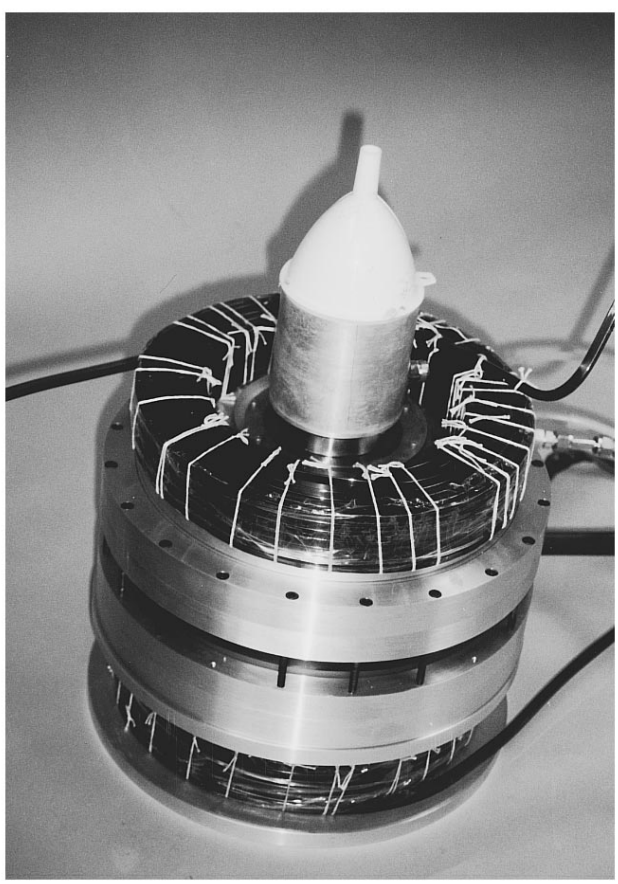

FIG. 7. A rf surface wave excited plasma source. Two dc magnetic coils provide a cusp field to confine plasma. A ring-shaped cathode is in the middle section. 
In order to explore broadening of the operating pressure window of the dc ring plasma ${ }^{2}$ we tried an auxiliary ICP discharge and a cusp shaped magnetic field separately. First, we combined the planar rf ICP discharge with a ring shaped cold cathode dc discharge, but the impedance of the dc discharge dropped by only $10 \%$ with the simple addition of rf. We then added an external cusp-shaped magnetic field to the ring discharge. The magnetic cusp field was generated by a pair of coils, each made from 42 turns of $6.35 \mathrm{~mm} \times 6.35$ $\mathrm{mm}$ copper wire, with a $17 \mathrm{~cm}$ inner diameter, a $26 \mathrm{~cm}$ outer diameter, and $4 \mathrm{~cm}$ in height. The space between the two coils was $16 \mathrm{~cm}$. A significant impedance reduction of the discharge resulted, allowing the disk plasma discharge to operate in a gas pressure region down to 2 mTorr.

Encouraged by this, we tried to replace the dc ring by rf. A surface wave launcher ${ }^{10}$ excited at $13.56 \mathrm{MHz}$ was used and was run with the dc magnetic cusp field shown in Fig. 7. A quartz tube of $3 \mathrm{~cm}$ in diameter was assembled with the surface wave launcher to couple $100 \mathrm{~W}$ of $13.56 \mathrm{MHz}$ rf power into the cusp field region. The diameter of the discharge vessel inbetween the coils was $21.5 \mathrm{~cm}$. The magnetic field at the center of the coil along the axis where the surface wave coupler was located was 190 gauss from a 100 A coil current, while 83 gauss of radial field was measured at the $21.5 \mathrm{~cm}$ edge of the symmetrical plate of the coil pair. Under these conditions the plasma glow was concentrated along the symmetrical plate and the axis of the system when the cusp field was applied. Oxygen, hydrogen, and argon at pressures of 1-100 mTorr were tested. Regardless of all these efforts with ring plasma, ${ }^{3}$ the planar ICP alone still gives superior performance for both hydrogen and oxygen.

\section{ACKNOWLEDGMENTS}

This work was supported by NSF Grant Nos. DDM9108531 and DDM-9311697, and by SEMATECH (Dr. Thuy Dao).

${ }^{1}$ J. S. Ogle, U.S. Patent No. 4948 458; Ch. Chen, D. Liu, and D. Tran, U.S. Patent No. 5226967.

${ }^{2}$ Z. Yu, Z. Luo, T. Sheng, H. Zarnani, Ch. Lin, and G. J. Collins, IEEE Trans. Plasma Sci. PS-18, 753 (1990).

${ }^{3}$ Z. Yu, T. Sheng, Z. Luo, and G. J. Collins, Appl. Phys. Lett. 57, 1873 (1990).

${ }^{4}$ N. Adachi, I. Serizawa, N. Ishibashi, D. Shaw, T. Sheng, and G. J. Collins, in Proceedings of the Fall Applied Physics Conference, Oct. 1991, Okayama, Japan.

${ }^{5}$ J. Hopwood, Appl. Phys. Lett. 62, 940 (1993).

${ }^{6}$ J. Hopwood, J. Vac. Sci. Technol. A 11, 147 (1993).

${ }^{7}$ M. S. Barnes, D. K. Coultas, J. Forster, J. Keller, U.S. Patent No. 5241245.

${ }^{8}$ Z. Yu, G. J. Collins, S. Hattori, D. Sugimoto, and M. Saita, Appl. Phys. Lett. 591194 (1991).

${ }^{9}$ D. Shaw, Z. Yu, D. Kobobel, G. J. Collins, and A. Takano, in Proceedings of AIP Conference No. 306, 1993, p. 538.

${ }^{10}$ M. Moisan and Z. Zakrzewski, J. Phys. D: Appl. Phys. 24, 1025 (1991). 